\title{
Diverse phenotypic consequences of mutations affecting the C-terminus of FLNA
}

\author{
Margriet van Kogelenberg • Alice R. Clark • Zandra Jenkins • \\ Tim Morgan • Ananda Anandan • Gregory M. Sawyer • Matthew Edwards • \\ Tracy Dudding • Tessa Homfray • Bruce Castle • John Tolmie • \\ Fiona Stewart • Emma Kivuva • Daniela T. Pilz • Michael Gabbett • \\ Andrew J. Sutherland-Smith • Stephen P. Robertson
}

Received: 29 September 2014 / Revised: 20 January 2015 / Accepted: 30 January 2015

(C) Springer-Verlag Berlin Heidelberg 2015

\begin{abstract}
Filamin A, the filamentous protein encoded by the X-linked gene FLNA, cross-links cytoskeletal actin into threedimensional networks, facilitating its role as a signalling scaffold and a mechanosensor of extrinsic shear forces. Central to these functions is the ability of FLNA to form V-shaped homodimers through its $\mathrm{C}$-terminal located filamin repeat 24. Additionally, many proteins that interact with FLNA have a binding site that includes the C-terminus of the protein. Here, a cohort of patients with mutations affecting this region of the protein is studied, with particular emphasis on the phenotype of male hemizygotes. Seven unrelated families are reported, with five exhibiting a typical female presentation of periventricular heterotopia $(\mathrm{PH})$, a neuronal migration
\end{abstract}

John Tolmie: In memoriam

Electronic supplementary material The online version of this article (doi:10.1007/s00109-015-1261-7) contains supplementary material, which is available to authorized users.

M. van Kogelenberg · Z. Jenkins · T. Morgan · S. P. Robertson $(\triangle)$ Department of Paediatrics and Child Health, Dunedin School of Medicine, Otago University, Dunedin, New Zealand

e-mail: stephen.robertson@otago.ac.nz

A. R. Clark · A. Anandan - G. M. Sawyer - A. J. Sutherland-Smith Institute of Fundamental Sciences, Massey University, Palmerston North, New Zealand

M. Edwards $\cdot$ T. Dudding

Hunter Genetics, Newcastle, Australia

\section{T. Homfray}

Department of Clinical Genetics, St George's Hospital, Tooting,

London, UK

B. Castle

Wessex Regional Genetics Service, Southampton, UK disorder typically caused by loss-of-function mutations in FLNA. One male presents with widespread PH consistent with previous male phenotypes attributable to hypomorphic mutations in FLNA. In stark contrast, two brothers are described with a mild $\mathrm{PH}$ presentation, due to a missense mutation (p.Gly2593Glu) inserting a large negatively charged amino acid into the hydrophobic dimerisation interface of FLNA. Co-immunoprecipitation, in vitro cross-linking studies and gel filtration chromatography all demonstrated that homodimerisation of isolated FLNA repeat 24 is abolished by this p.Gly2593Glu substitution but that extended FLNA $^{\text {Gly2593Glu }}$ repeat 16-24 constructs exhibit dimerisation. These observations imply that other interactions apart from those mediated by the canonical repeat 24 dimerisation

\footnotetext{
J. Tolmie

Fergusson-Smith Centre for Clinical Genetics, Glasgow, UK

F. Stewart

Clinical Genetics Service, Belfast City Hospital, Belfast, UK

E. Kivuva

Clinical Genetics, Royal Devon and Exeter Hospital, Exeter, UK

D. T. Pilz

Institute of Medical Genetics, University Hospital of Wales, Cardiff

University, Cardiff, UK

M. Gabbett

Genetic Health Queensland, Royal Brisbane and Women's Hospital and School of Medicine, Griffith University, Gold Coast, Australia
} 
interface contribute to FLNA homodimerisation and that mutations affecting this region of the protein can have broad phenotypic effects.

\section{Key messages}

- Mutations in the X-linked gene FLNA cause a spectrum of syndromes.

- Genotype-phenotype correlations are emerging but still remain unclear.

- C-term mutations can confer male lethality, survival or connective tissue defects.

- Mutations leading to the latter affect filamin dimerisation.

- This deficit is compensated for by remotely acting domains elsewhere in FLNA.

Keywords Filamin A · Periventricular neuronal heterotopia . Dimerisation

\section{Introduction}

The actin binding protein filamin A (FLNA) plays a key role in human development, and mutations are causative of several human conditions including disorders of neuronal migration, skeletal development and cardiac valvular structure [1-5]. FLNA, encoded by the X-linked gene, FLNA, is nearubiquitously expressed and is highly efficient at crosslinking actin into three-dimensional networks [6]. Additional interactions with transmembrane proteins and cell surface receptors implicate FLNA as an adaptor protein that links the cell membrane to the cytoskeleton in both a mechanical and functional sense [7-9]. The cytoskeletal cross-linking and scaffolding functions of FLNA are reflected in its structure which is characterised by a conserved $\mathrm{N}$-terminal actin binding domain (ABD) followed by $24 \beta$-pleated 'filamin repeats' that exhibit significant structural homology (Fig. 1a) [10]. Interposed within this array of filamin repeats are two hinge regions located between repeat 15-16 (hinge 1) and 23-24 (hinge 2) [11] that confer flexibility to the filamin rod [12]. Dimerisation of filamin is mediated by repeat 24 at the Cterminus of the protein $[11,13,14]$.

The ability of FLNA to effectively cross-link actin is mediated by the Y-shaped orientation of the protein dimer anchored by the dimerisation domain within repeat 24 and further facilitated by the flexibility conferred by the two hinge regions (Fig. 1a) [6, 12, 15]. This arrangement also contributes to cellular mechanoprotection upon the application of extrinsic forces [11, 16-19]. Removal of the repeat 24 dimerisation domain disrupts these properties in polymerised actin gels in vitro $[11,12,20]$, and the specific interposition of charged residues into the hydrophobic dimerisation interface of the highly homologous repeat 24 domain in FLNC [15, 21] also results in the loss of dimerisation [15]. Consequently, the $\mathrm{C}$ terminal dimerisation function of FLNA has been considered to be indispensable for successful development as reflected by the male-lethal phenotypes conferred by null alleles at this locus $[11,13,14,20]$.

Heterozygosity for null FLNA alleles leads to the neurological disorder periventricular neuronal heterotopia (PH). $\mathrm{PH}$ is characterised by nodules of grey matter abutting the cerebral ventricular walls. The nodules represent neurons that have failed to undertake radial neuronal migration to the cerebral cortex during fetal development. Due to the $\mathrm{X}$ chromosomal location of the gene, FLNA mutations causing $\mathrm{PH}$ are generally embryonic lethal in hemizygous males with survival usually attributed to hypomorphic or mosaic mutations resulting in some retained FLNA function [22-26].

Here, we present a series of mutations occurring in exons 47 and 48 of FLNA that encode repeat 24 of FLNA. Understanding the phenotypic consequences of mutations in this region of the protein is important since the mutations could conceivably affect the stability of the protein, its ability to scaffold interacting proteins or its ability to sponsor dimerisation. In six unrelated individuals, both missense and frameshift mutations have led to a typical presentation of $\mathrm{PH}$ with one female conceiving a male fetus with extensive bilateral PH consistent with some residual retained FLNA function associated with her mutant allele. In a seventh family however, two related males with unusually mild $\mathrm{PH}$ are shown to be hemizygous for a missense mutation, inherited from their nonpenetrant mother, that predicts a Gly $\rightarrow$ Glu substitution within repeat 24 . This mutation leads to significant impairment of canonical repeat 24-mediated FLNA homodimerisation in vitro, but this loss may be compensated by an alternative mechanism, mediated either directly or indirectly via the $\mathrm{C}$ terminal FLNA rod region. This compensatory activity restores at least partial FLNA function that possibly explains this mild phenotype in this family and in another unrelated female with the same mutation reported elsewhere [27].

\section{Material and methods}

Human subjects and FLNA mutation screening

Participants in the study were consented through a protocol approved by the Otago Ethics Committee in accordance with the principles laid out in the Declaration of Helsinki. The exon and intron-exon boundaries of FLNA were amplified by PCR using previously published primers and sequenced on an ABI3100 capillary sequencer [3]. All mutations were independently verified by repeat sequencing on independent PCR-amplified products. All mutations in FLNA refer to 
a

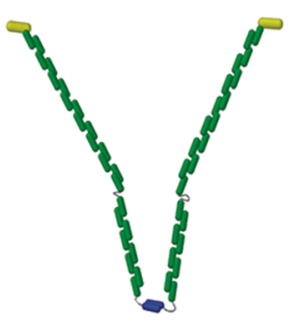

b

$$
\begin{aligned}
& \text { Hinge } 2 \text { | VSNHSLHETSSVFVDSLTKATCAPQHGAPGPGP } 2554 \\
& \text { ADASKVVAKGLGLSKAYVGQKSSFTVDCSKAGN } 2587 \\
& \begin{array}{c|c}
7 & \\
\text { Repeat } 24 & \text { NMLLVĞVHGPRTPCEEILVKHVGSRLYSVSYLL } 2620
\end{array} \\
& \text { KDKGEYTLVVKWGDE } \underset{3}{\mathbf{3}} \text { EHIPGSPYRVVVP }
\end{aligned}
$$

e

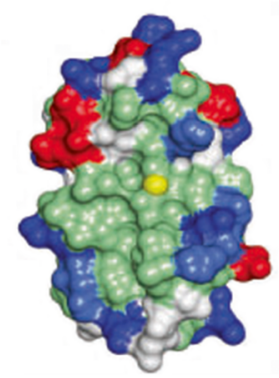

f

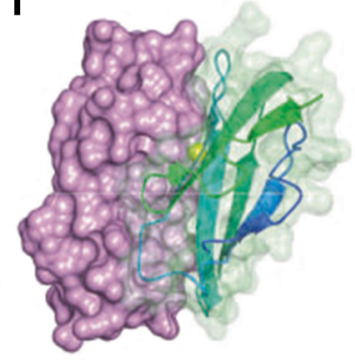

Fig. 1 Schematic overview of FLNA, mutations and clinical presentation of the cohort and structural consequences of the p.Gly2593Glu substitution within repeat 24. a Schematic of dimeric FLNA. The yellow regions represent the $\mathrm{N}$-terminal actin binding domain. The rod domain, depicted in green, is composed of 24 filamin repeats, with repeat 24 (blue) mediating dimerisation through the anti-parallel apposition of the two repeat 24 domains. The rod domain is interrupted by two hinge regions between repeats 15 and 16 (hinge 1) and repeats 23 and 24 (hinge 2). b Amino acid sequence of the C-terminus of FLNA. Residues constituting hinge 2 are presented in the top line with the sequence of repeat 24 in the three subsequent rows. Sequences encoded by exon 47 (blue) and 48 (red) are indicated with numbers $1-7$ (Table 1) indicating the position of mutations (underlined red residues). c Axial MRI scans of the brain from the index male from case 7 with a single heterotopic grey matter nodule indicated by the circle (left) and the mother from family 6 showing diffuse heterotopic grey matter lining the lateral walls of the ventricles bilaterally (right). d Immunocytochemistry of cultured primary dermal

Genbank accession number NM_001110556.1, and the protein coordinates to NP_001104026.1

\section{Immunofluorescence}

Human fibroblasts were fixed with $1 \%$ paraformaldehyde, blocked and permeabilised with $7 \%$ fetal calf serum (FCS), $1 \%$ Triton X100 in PBS and incubated with the primary then secondary antibodies prior to counterstaining with DAPI 1:5000. Cells were visualised on a Zeiss Axioplan microscope fitted with a (Slider-6, $1360 \times 1024,1.39$ megapixel) CCD camera, and images were captured using Spot Advanced analysis software version 4.6 (Diagnostic Instruments, Sterling Heights, MI, USA).

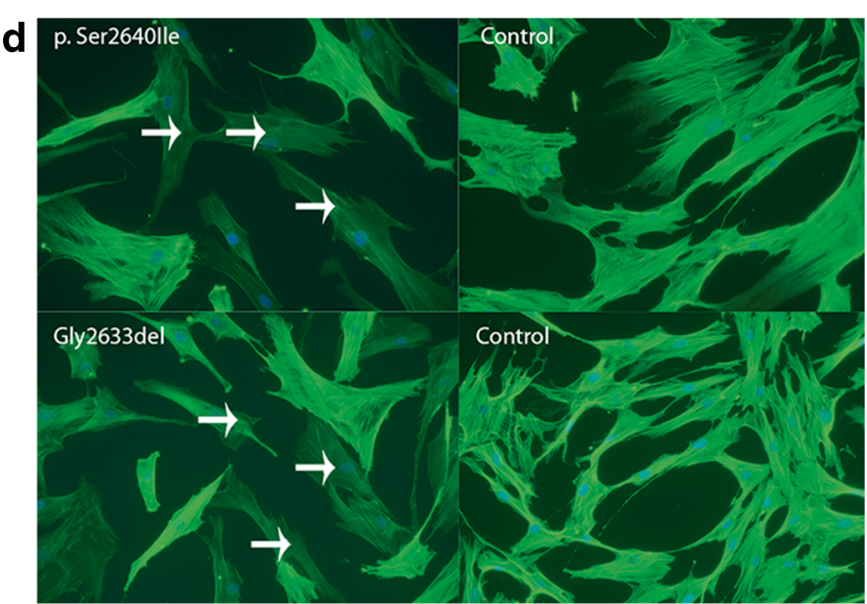

g

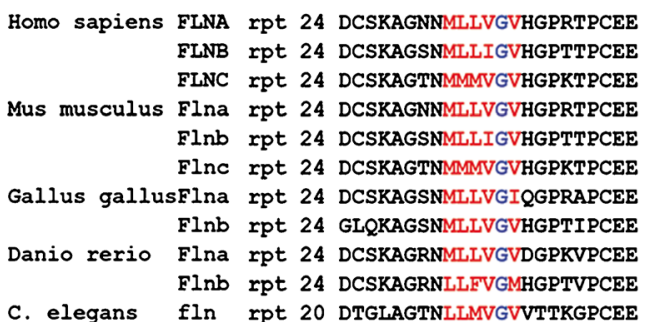

fibroblasts from cases 3 and 5, both of whom are females with a random patterns of $\mathrm{X}$ inactivation (data not shown). In each culture, a more faintly staining population of cells can be observed (arrows) that is absent in control cultures. e Structure of the FLNA_rpt $24^{\mathrm{WT}}$ dimerisation interface (en face; one monomer shown for clarity). Gly2593 (C $\alpha)$, that is substituted in case 7 is highlighted in yellow, and the domain surface is colour coded by amino acid property; hydrophobic green, polar uncharged white, acidic red, basic dark blue and histidine light blue. f Structure of the FLNA_rpt $24^{\mathrm{WT}}$ dimer showing the position of Gly 2593 (yellow), $\mathrm{C} \alpha$ from one monomer (green) buried against its partner monomer (purple) at the dimer interface. A ribbon representation ( $\mathrm{N}$-terminus dark blue) of the green monomer is shown under the transparent surface. g Amino acid sequence alignment of the dimerisation interface in several species indicating complete conservation of the substituted residue Gly2593 (blue) within the sequence that constitutes the anti-parallel $\beta$ sheet hydrophobic dimerisation interface (red)

Fibroblast migration assay

Motility assays were carried out as described [28]. Tissue culture plates were coated overnight $\left(4^{\circ} \mathrm{C}\right)$ with fibronectin $(10 \mu \mathrm{g} / \mathrm{ml})$ in PBS. Unbound fibronectin was removed; the plates rinsed with PBS and blocked with BSA at $37^{\circ} \mathrm{C}$ for $1 \mathrm{~h}$. The plates were further rinsed with PBS, and Dulbecco's modified Eagle's medium (DMEM) plus $20 \%$ FCS was then added followed by seeding with fibroblasts $\left(5.0 \times 10^{4}\right)$ which were grown for 3 days until $90 \%$ confluent when the plate was scratched with a pipette tip. The media was replaced $(10 \%$ FCS $)$ and cells incubated at $37{ }^{\circ} \mathrm{C}$ with $5 \% \mathrm{CO}_{2}$. The width of the scratch was measured by light microscopy at various time points and captured images analysed using ImageJ [29]. The change in width of the scratch divided by 
the time incubated gave the rate $(\mathrm{mm} / \mathrm{h})$. The experiments were conducted in triplicate.

\section{Western blotting}

Fibroblasts were grown to $90 \%$ confluence in DMEM media, washed with PBS and lysed $(10 \mathrm{mM}$ Tris/ $\mathrm{HCl}, \mathrm{pH} 7.4$, $100 \mathrm{mM} \mathrm{NaCl}, 1 \mathrm{mM}$ EDTA, $1 \mathrm{mM}$ EGTA, $1 \%$ Triton $\mathrm{X}-100,10 \%$ glycerol, $0.1 \%$ sodium dodecyl sulfate (SDS), $0.5 \%$ deoxycholate, protease inhibitor). The cell lysate was centrifuged; the supernatant diluted to $0.5 \mathrm{mg} / \mathrm{ml}$ protein concentration and run on $10 \%$ SDS-polyacrylamide gel electrophoresis (PAGE). Overnight transfer to nitrocellulose was conducted in transfer buffer $(25 \mathrm{mM}$ glycine, $20 \mathrm{mM}$ Tris/HCl, $7.6 \mathrm{mM}$ SDS, $20 \%$ methanol) and confirmed by Ponceau stain. The nitrocellulose was blocked with $5 \% \mathrm{w} / \mathrm{v}$ skim milk powder in Tris-buffered saline with Tween 20 (TBST; $20 \mathrm{mM}$ Tris/HCl, $150 \mathrm{mM} \mathrm{NaCl}, 0.05 \%$ Tween 20, $\mathrm{pH}$ 7.5) for $1 \mathrm{~h}$, rinsed with TBST and incubated with either anti-FLNA (Chemicon MAB1678, 1:15,000) or anti- $\alpha$ tubulin antibody (Sigma T9026, 1:4000) for $1.5 \mathrm{~h}$. After washing, the membranes were incubated with mouse secondary antibody (Sigma) diluted to 1/6000 in TBST, washed thoroughly with TBST and detected using the SuperSignal West Pico solution (Pierce) in an intelligent dark box II (Fujifilm). Densitometry was performed with the LAS-1000 pro software.

\section{Construct design}

Expression constructs were designed in the pcDNA3.1 expression vector (Invitrogen) and p3xFLAG-CMV-7.1 expression vector (Sigma-Aldrich). The c.7778 G>A FLNA mutation was introduced by site-specific mutagenesis with either an N-terminal FLAG or C-terminal V5 tag. For Escherichia coli overexpression, FLNA repeat domain 24 (residues 25552648) complementary DNA (cDNA) was generated by PCR using Pwo DNA polymerase (Roche) and full-length FLNA cDNA as template. The amplified product was digested and ligated into the BamHI and XhoI restriction sites of the expression vector pProEXHTb (Invitrogen). The Gly2593Glu mutant was prepared by mutagenesis PCR using Pfu Turbo high fidelity DNA Polymerase (Stratagene).

Cellular transformation, expression

and co-immunoprecipitation

HEK293T cells were grown in DMEM supplemented with $10 \%$ fetal bovine serum at $37{ }^{\circ} \mathrm{C}$ in $5 \% \mathrm{CO}_{2}$. Expression constructs were transiently transfected in HEK293T cells using Lipofectamine ${ }^{\mathrm{TM}} 2000$ (Invitrogen). Cells were lysed after $20 \mathrm{~h}$ in $1 \times$ PBS, $1 \%$ Triton X100 and complete protease inhibitor (Roche) and either run directly on SDS-PAGE or subject to co-immunoprecipitation using mouse anti-V5 and Protein G Sepharose (Invitrogen). Immunoprecipitated complexes were washed in lysis buffer, transferred to nitrocellulose membrane (Pierce) and probed with mouse anti-FLAG (Sigma) and/or mouse anti-V5 (Invitrogen) antibodies followed by detection with Supersignal West Pico (Pierce). FLNA_rpt $24^{\mathrm{WT}}$ and FLNA_rpt2 ${ }^{\mathrm{G} 2593 \mathrm{E}}$ proteins were overexpressed in E. coli BL21 (DE3) grown in LuriaBertani (LB) broth supplemented with $100 \mu \mathrm{g} / \mathrm{ml}$ ampicillin. Single colony cultures were grown at $37^{\circ} \mathrm{C}$ and induced with $200 \mu \mathrm{M}$ isopropyl- $\beta$-D-thiogalactoside (IPTG) when $\mathrm{A}_{600}$ reached $0.6-0.8$. The cultures were transferred to $25^{\circ} \mathrm{C}$ and grown in an orbital shaker for $16 \mathrm{~h}$ followed by harvesting by centrifugation and stored frozen. The cells were lysed in $20 \mathrm{mM}$ Tris (pH 8.0), $500 \mathrm{mM} \mathrm{NaCl}, 20 \mathrm{mM}$ imidazole and passed through a French Press twice at 5000 psi and further sonicated. The supernatant, after centrifugation at $30,000 \times g$ for $20 \mathrm{~min}$, was applied to a $5 \mathrm{ml} \mathrm{Ni}^{2+}$-NTA HisTrap column (GE Healthcare), and the histidine-tagged FLNA24 domain eluted in the same buffer with 500-600 mM imidazole. The repeat domains were purified further on a Superdex $75 \mathrm{HR} 30 /$ 10 gel filtration column in buffer $20 \mathrm{mM} \mathrm{K} \mathrm{HPO}_{4} / \mathrm{KH}_{2} \mathrm{PO}_{4}$, pH 7.0, $80 \mathrm{mM} \mathrm{NaCl}, 5 \mathrm{mM}$ dithiothreitol (DTT), $1 \mathrm{mM}$ $\mathrm{MgCl}_{2}$. FLNA rpt24 ${ }^{\mathrm{WT}}$ eluted as a single dimer peak while FLNA_rpt $24^{\mathrm{G}} \overline{2593 \mathrm{E}}$ eluted as a smaller molecular weight species consistent with a monomeric population [15].

FLNA repeat 24 domain dimerisation cross-linking assay

FLNA repeat 24 cross-linking experiments were conducted as described [13]. In brief, FLNA repeat 24 proteins of final concentration $50 \mathrm{ug} / \mathrm{ml}\left(20 \mathrm{mM} \mathrm{K} \mathrm{HPO}_{4} / \mathrm{KH}_{2} \mathrm{PO}_{4}, \mathrm{pH} 7.0\right.$, $80 \mathrm{mM} \mathrm{NaCl}, 1 \mathrm{mM}$ DTT, $1 \mathrm{mM} \mathrm{MgCl}{ }_{2}$ ) were incubated with 0-8 $\mathrm{mM}$ ethylene glycol bis(succinimidylsuccinate) (EGS; previously dissolved in dimethyl sulfoxide (DMSO)) or just the buffer/DMSO components alone without EGS $(0 \mathrm{mM}$ control). The samples were incubated at $37^{\circ} \mathrm{C}$ for $20 \mathrm{~min}$. The cross-linking reaction was stopped by trichloroacetic acid precipitation (40\%) on ice for $30 \mathrm{~min}$. The pellets were collected by centrifugation $12,000 \times g$ for $15 \mathrm{~min}$, washed with ice-cold acetone and air dried. The protein pellets were resuspended in SDS-PAGE loading buffer, heat denatured and analysed by SDS-PAGE.

\section{Results}

Clinical synopses and mutation analysis

As part of a study on the molecular pathology of X-linked $\mathrm{PH}$, seven probands were identified with mutations occurring within the two most $3^{\prime}$ exons (exons 47 and 48) of FLNA, which encode the hinge 2 and repeat 24 domains of FLNA 
(Fig. 1; Table 1). The clinical manifestations in affected females in families 1-6 were typical for X-linked PH; extensive regions of periventricular nodular grey matter were noted along the lateral borders of the bodies of the lateral cerebral ventricles that were seen in all individuals. Family 7 , however, was atypical in that the two affected individuals were brothers and their mother, who was heterozygous for their mutation, was clinically and radiologically non-penetrant for the disorder.

Five separate mutations were identified in this cohort. Two were recurrent in unrelated families, one predicting a frameshift (c.7612delCT) and one an in-frame deletion of a single amino acid (c.7897del3). Single instances of two missense mutations (c.7919G $>$ T, c. $7778 \mathrm{G}>\mathrm{A}$ ) and a frameshift (c.7900delG) were also noted. The recurrent truncating mutation in exon 47, c.7612delCT, is predicted to terminate protein translation at the end of hinge 2 and produce a protein entirely lacking repeat 24 or alternatively to trigger nonsense mediated mRNA decay and constitute a null allele (Fig. 1b). The effect of the recurrent mutation c.7897del3 on protein expression was difficult to predict, and therefore, cultured skin fibroblasts from case 3 , a female with a random pattern of $\mathrm{X}$ inactivation in this tissue, were subject to immunostaining with an antiFLNA antibody. Two distinct populations of cells were observed, one staining less intensely for cytoskeletal FLNA, suggesting retained expression from the mutant allele but with reduced protein stability (Fig. 1c). Similar results were obtained for fibroblasts obtained from the individual who was heterozygous for the p.Ser2640Ile mutation (case 5). Collectively, cases $1-5$ indicate that both truncating and missense mutations affecting the very C-terminus of FLNA usually manifest as typical female-limited $\mathrm{PH}$. The retention of some protein expression from the mutant allele (Fig. 1c) is consistent however with male survivorship since similar levels have been observed in surviving males previously [30].

Mutations in FLNA in males (families 6 and 7) are thought to only result in survival if they are hypomorphic or are present in a mosaic state. This male phenotype typically comprises
PH distributed in a fashion very similar to that seen in female heterozygotes with null mutations. The mutation in family 6 resulted in a classic PH phenotype in the mother (Fig. 1d). A mid-gestation fetal ultrasound scan during pregnancy demonstrated dilated cerebral ventricles, and a fetal MRI showed bilateral PH in a male fetus. The pregnancy was medically terminated, and at post mortem, dense contiguous sheets of heterotopic neurons were observed histologically. In contrast, the mutation in family 7 produced much milder manifestations in males. The proband presented with sudden left-sided sensorineural hearing loss in childhood prompting neuroimaging studies. There was a history of migraines and infrequent generalised seizures. His neurodevelopment was normal, and his scholastic performance was above average. Cerebral MRI scan demonstrated a single subependymal grey matter heterotopion at the margin of the right ventricle (Fig. 1c). Subsequent study of the younger brother of the proband revealed three nodules of neuronal heterotopia. He had a history of a single seizure at 10 months of age but no history of deafness. Both the proband and his brother had a tall thin habitus, retinal lattice degeneration on fundoscopy, tall stature, joint hypermobility and high arched palates. Echocardiography demonstrated normal cardiac structure and function.

Sequence analysis of FLNA revealed a hemizygous missense variant (c.7778G $>$ A; p.Gly2593Glu) in the two brothers. The mother was heterozygous for the same variant but was clinically normal, and no PH was observable on MRI. FLNA sequence analysis on the maternal grandmother detected no mutations, but the maternal grandfather was unavailable for study. The c. $7778 \mathrm{G}>\mathrm{A}$ variant was neither observed in dbSNP or the 1000 genomes database (www.1000genomes. org). The p.Gly2593Glu substitution positions a large negatively charged amino acid in the hydrophobic region that constitutes the repeat 24 dimerisation interface [21] (Fig. 1e-g). The substituted Gly2593 is highly conserved among filamin isoforms and across species (Fig. 1g). Further supporting its pathogenicity is the observation of the same
Table 1 Sequences encoded by exon 47 and 48

\begin{tabular}{lllll}
\hline Case & Gender & Mutation & Predicted protein & Phenotype \\
\hline 1 & F & c.7612delCT & p.Leu2538Aspfs*18 & Bilateral PH \\
2 & F & c.7612delCT & p.Leu2538Aspfs*18 & Bilateral PH \\
3 & F & c.7897del3 & p.2633delGly & Bilateral PH \\
4 & F & c.7897del3 & p.2633delGly & Bilateral PH \\
5 & F & c.7919G $>$ T & p.Ser2640Ile. & Bilateral PH \\
6 & F (mother) & c.7900delG & p.Asp2634Thrfs*71 & Bilateral PH \\
& M & c.7900delG & p.Asp2634Thrfs*71 & Bilateral PH \\
7 & F (mother) & c.7778G $>$ A & p.Gly2593Glu & Normal MRI \\
& M & c.7778G $>$ A & p.Gly2593Glu & Unilateral paucinodular PH \\
& M & c.7778G $>$ A & p.Gly2593Glu & Unilateral paucinodular PH \\
\hline
\end{tabular}

$F$ female, $M$ male, $P H$ periventricular neuronal heterotopia 
mutation arising de novo in an unrelated female with paucinodular $\mathrm{PH}$ [27].

The functional importance of Gly2593 is also indicated by site-directed mutagenesis studies of residues within the dimerisation interface of the highly homologous repeat 24 of FLNC. Introduction of a p.Met2669Asp substitution resulted in the loss of a hydrophobic interaction with Gly2671 of the adjacent monomer [15]. In FLNA, Gly2593 is the equivalent of FLNC Gly2671 implying that substitution of Gly2593 would similarly disrupt FLNA homodimerisation. Together, these data implicate the c. $7778 \mathrm{G}>\mathrm{A}$ mutation as causative of the observed PH phenotype, although the mildness of the presentation is paradoxical in the face of the predicted molecular consequences of this substitution in disrupting dimerisation between FLNA monomers.

\section{FLNA expression in the proband}

To further characterise the cellular phenotype associated with the clinical presentation of the males from family 7 , the subcellular localisation of FLNA and FLNB in cultured dermal fibroblasts was assessed by immunohistochemistry. The distribution of both FLNA and FLNB in cells from the proband was indistinguishable from control cells (Fig. 2a). Since FLNA-deficient human melanoma cell lines demonstrate impaired locomotion [31], a scratch migration assay was performed to determine the locomotor capabilities of patient cells on fibronectin-coated slides. This assay demonstrated that the motility of cells hemizygous for the p.Gly2593Glu substitution was unimpaired (Fig. 2b, d). Western analysis of fibroblasts obtained from the proband indicated no change in FLNA protein levels as a consequence of this mutation (Fig. 2c-d). These data suggest that FLNA function is substantially preserved in cells that possess this substitution in the dimerisation domain, implying that either the mutation does not disrupt dimerisation in the manner previously demonstrated for an equivalent substitution in FLNC [15] or that the dimerisation function is compensated for by an unrecognised mechanism.

\section{FLNA $^{\text {G2593E }}$ repeat 24 cannot homodimerise}

To test whether the dimerisation interface is disrupted by the c. $7778 \mathrm{G}>$ A mutation, expressed proteins containing this mutation were studied in purified form in vitro and also within their cellular context. Chemical covalent cross-linking studies performed on bacterially expressed FLNA repeat 24 with (FLNA_rpt24 ${ }^{\mathrm{G} 2593 \mathrm{E}}$ ) and without (FLNA_rpt24 ${ }^{\mathrm{WT}}$ ) the substitution showed that the Gly2593Glu alteration dramatically inhibits FLNA repeat 24 dimerisation (Fig. 3a). Circular dichroism studies indicated that FLNA_rpt $24^{\text {WT }}$ and FLNA_rpt24 $4^{\text {Gly2593Glu }}$ proteins adopted broadly equivalent $\beta$-sheet-rich structures showing that the reduced ability of the mutant to dimerise is not due to gross misfolding, although FLNArpt24 ${ }^{\text {Gly2593Glu }}$ did exhibit reduced thermal stability at elevated temperatures $\left(>50^{\circ} \mathrm{C}\right.$; data not shown). Analytical gel filtration chromatography studies using these same proteins showed that the FLNA rpt $24^{\mathrm{WT}}$ protein eluted significantly earlier compared to FLNA_rpt24 ${ }^{\mathrm{G} 2593 \mathrm{E}}$, consistent with the wild-type protein forming dimeric structures and hence exhibiting a smaller elution volume (Fig. 3b). These results closely paralleled those obtained for site-directed mutations in FLNC repeat 24 that disrupt the dimerisation interface of this filamin paralogue $[13,15]$.

Since FLNA_rpt24 ${ }^{\mathrm{G} 2593 \mathrm{E}}$ cannot homodimerise in cell-free systems and mindful that previous studies have asserted the primacy of repeat 24 in the mediation of dimerisation by employing similar experimental systems [13, 20], corroborative evidence for disruption of homodimerisation was sought using FLNA constructs expressed in a cellular context. The ability of FLNA ${ }^{\mathrm{G} 2593 \mathrm{E}}$ repeat 24 to homodimerise was examined using transfection and co-expression of FLAG- and V5tagged minigene constructs in HEK293 cells. Constructs were designed to express FLNA repeat 24 wild type (FLNA_rpt24 ${ }^{\mathrm{WT}}$ ) or Gly2593Glu (FLNA_rpt24 ${ }^{\mathrm{G} 2593 \mathrm{E}}$ ), fused to either N-terminal FLAG or C-terminal V5 tags, in HEK293 cells and co-immunoprecipitated using either anti-V5 or antiFLAG antibodies. FLNArpt $24{ }^{\text {WT }}$ was demonstrated to homodimerise (Fig. 4a). Analogous co-IP experiments with the FLNA_rpt24 ${ }^{\mathrm{G} 2593 \mathrm{E}}$ constructs demonstrated that FLAGtagged FLNA ${ }^{\mathrm{G} 2593 \mathrm{E}}$ repeat 24 did not co-precipitate with a V5-tagged FLNA ${ }^{\text {G2593E }}$ repeat 24 (Fig. 4a). Reciprocal tag swapping experiments produced identical results (Fig. 4a). Overexposure of the immunoblot did detect small amounts of both the anti-V5 and anti-FLAG fusion proteins, indicating that the interaction is substantially, but not completely, abolished compared to FLNA_rpt24 ${ }^{\text {WT }}$ (Supplementary Fig. 1).

A compensatory dimerisation mechanism is mediated by repeats $16-23$ of filamin A

Demonstrating defective dimerisation of FLNA ${ }^{\mathrm{G} 2593 \mathrm{E}}$ repeat 24 using both biochemical and cellular approaches was surprising in light of the mild clinical phenotype in the males in family 7 , suggesting that this mutation does not lead to complete loss of FLNA function. Since repeat 24 has been shown to be sufficient for dimeric FLNA cross-linking activity [13], a compensatory mechanism could explain this apparent retention of partially functional FLNA. One possibility is the facilitation of homodimerisation by protein-protein interactions involving FLNA and the C-terminal rod region since previous microscopic and cross-linking studies using purified recombinant FLNA constructs in vitro have not demonstrated dimerisation in the absence of intact repeat $24[11,20]$. To further dissect the dimerisation capabilities of FLNA ${ }^{\mathrm{G} 2593 \mathrm{E}}$ 


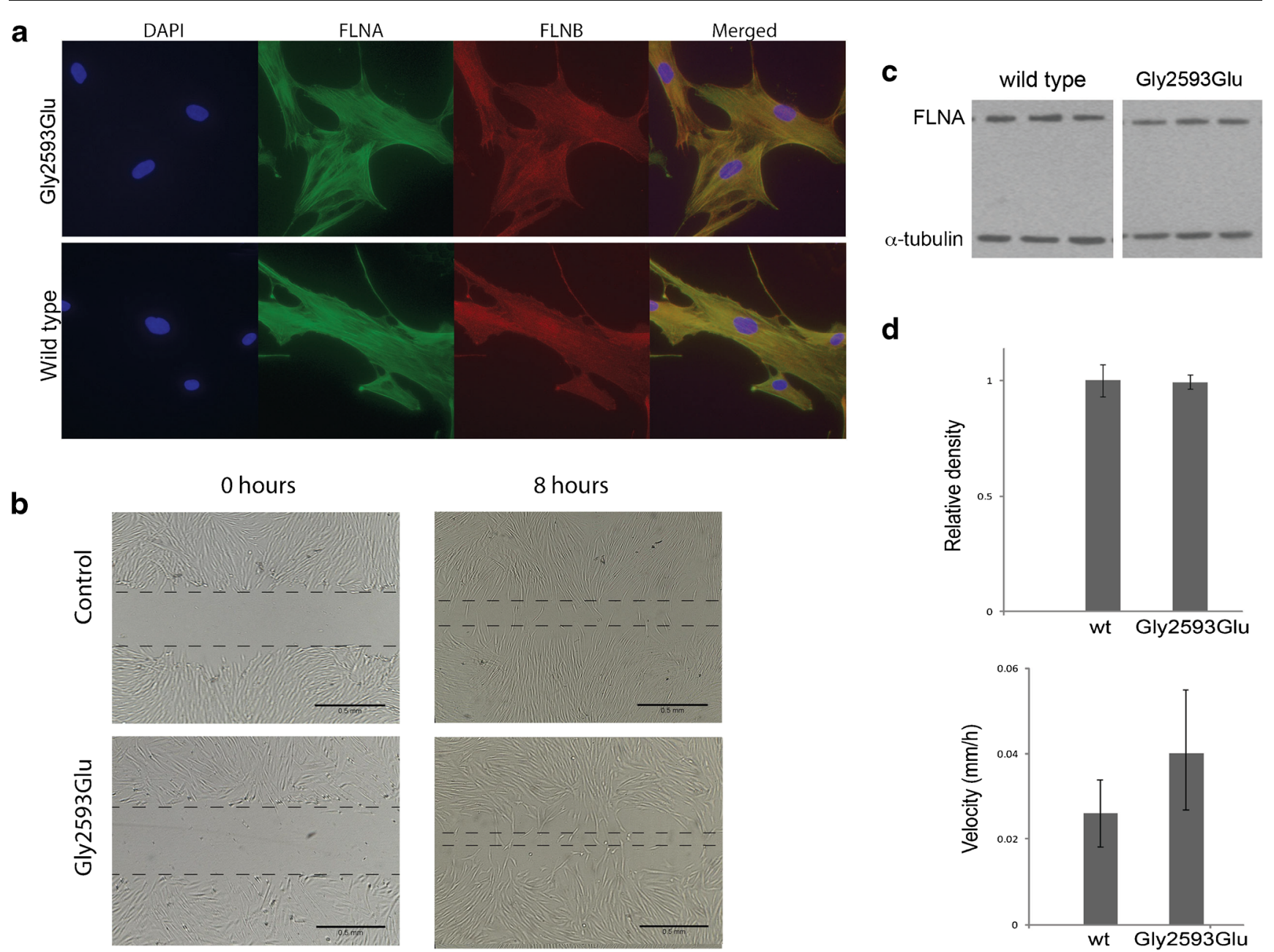

Fig. 2 The Gly2593Glu substitution does not affect FLNA expression or cell motility. a Indirect immunofluorescence images of cultured skin fibroblasts from the proband and a control individual. DAPI (blue), FLNA (green) and FLNB (red). b Phase contrast light microscopy images of cell migration performed on confluent cultures of primary fibroblasts with and without the Gly2593Glu substitution in FLNA.
Images from $t=0$ and $8 \mathrm{~h}$ are shown. $\mathbf{c}$ Western blot analysis of endogenous FLNA protein in skin fibroblast cells from the proband compared to control and normalised to $\alpha$-tubulin. d Graphical representation of results from western analysis (top) and the migration assay (lower) tagged constructs containing hinge 1 and repeats 16-24 (FLNA_rpt16-24 ${ }^{\mathrm{WT}}$ and FLNA_rpt16-24 ${ }^{\mathrm{G} 2593 \mathrm{E}}$ ) were generated, co-transfected into HEK293 cells and coimmunoprecipitated using either anti-V5 or anti-FLAG antibodies. In contrast to experiments employing repeat 24 in isolation, both FLNA_rpt16-24 ${ }^{\mathrm{WT}}$ and FLNA_rpt16$24^{\mathrm{G} 2593 \mathrm{E}}$ did homodimerise (Fig. 4b). Both the co-IP of FLNA $^{\mathrm{WT}}$ and FLNA ${ }^{\mathrm{G} 2593 \mathrm{E}}$ containing proteins demonstrated a similar capacity to form homodimers.

\section{Discussion}

Repeat 24 of FLNA is central to the mechanosensory and scaffolding functions of this protein. Here, a cohort of individuals is described with germline mutations affecting this protein domain and leading to variable phenotypic presentations. Consistent with previous findings, the phenotypes observed in the majority of instances were those of femalelimited bilateral PH. Interestingly, some of these mutations are associated with reduced, but not absent, expression of FLNA protein from the mutant allele, an observation that is consistent with some retained function of FLNA. It is conceivable that missense mutations or even nonsense mutations (because their $3^{\prime}$ location in the gene means that they can escape nonsense mutation-mediated transcript decay) that affect repeat 24 could still be compatible with survival in males. This possibility is realised in the cohort reported here in family 6 where a missense mutation leads to a presentation of $\mathrm{PH}$ in both the heterozygote and her male fetus. Other mutations reported here in only the heterozygous state could conceivably confer survivability in males given evidence for diminished 


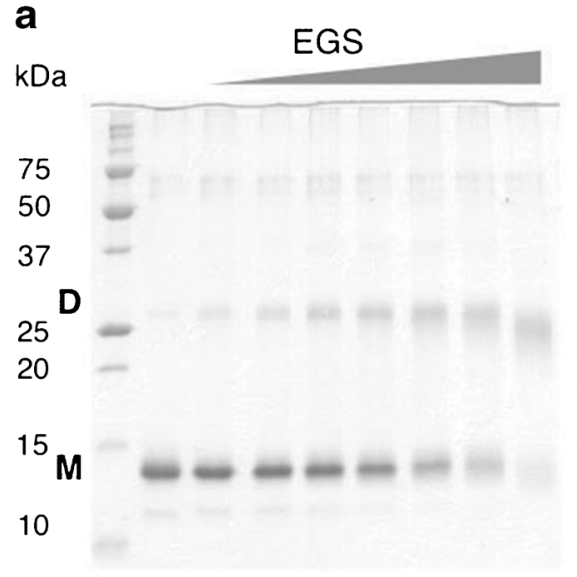

FLNA_rpt24WT

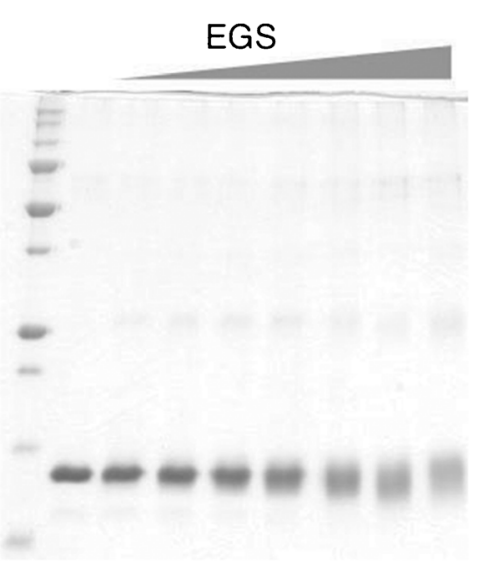

FLNA_rpt24 ${ }^{\text {G2593E }}$ b

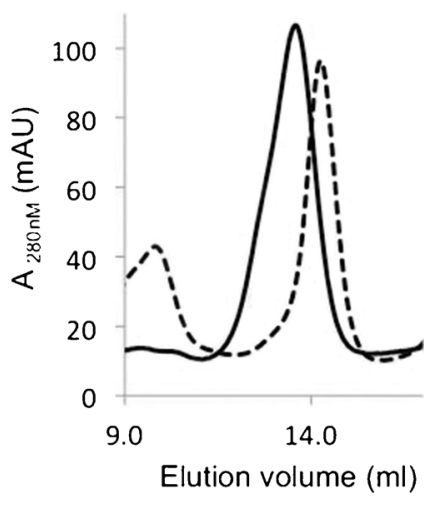

Fig. 3 FLNA rpt24 $4^{\mathrm{Gly} 2593 \mathrm{Glu}}$ does not homodimerise in vitro. a Ethylene glycol bis(succinimidylsuccinate) (EGS) covalent cross-linking. SDS-PAGE gels of FLNA_rpt24 ${ }^{\mathrm{WT}}$ (left) and FLNA_rpt24 ${ }^{\mathrm{G} 2593 \mathrm{E}}$ (right). Repeat 24 domains were incubated in increasing concentrations of EGS: $0,0.05,0.1,0.2,0.4,0.8,1.6,8.0 \mathrm{mM}$ at room temperature for $30 \mathrm{~min}$ before TCA precipitation and SDS-PAGE analysis. The proportion of covalently cross-linked FLNA_rpt24 ${ }^{\mathrm{G} 2593 \mathrm{E}}$ dimer (D), relative to monomer (M), is greatly reduced at higher EGS concentrations compared to FLNA rpt $24^{\mathrm{WT}}$. b Gel filtration chromatography elution profiles of FLNA_rpt24 ${ }^{\mathrm{WT}}$ (solid line) and FLNA_rpt24 ${ }^{\mathrm{G} 2593 \mathrm{E}}$ (dashed line)

functions mediated by this protein. The paradoxical observation of mild PH observed in two males, with non-penetrance in their mother, in the context of a FLNA mutation that abolishes canonical dimerisation via repeat 24 , directly implies significant retention of FLNA functionality. The heterozygous mother in case 7 is clinically and neuroradiologically non-
Fig. 4 Homodimerisation of FLNA rp16-24 ${ }^{\text {Gly2593Glu }}$ but not FLNA rpt24 Gly2593Glu. a HEK 293 cells co-transfected with FLAG-tagged FLNA rpt24 Gly2593Glu and V5-tagged FLNA_rpt $24^{\text {Gly2593Glu }}$. After $12 \mathrm{~h}$ growth cell lysates subject to immunoprecipitation using antiFLAG or anti-V5 antibodies linked to agarose beads and analysed by Western blot. b Results from HEK293 cells cotransfected with FLAG-FLNA rpt16-24 Gly2593Glu and V5FLNA rpt16-24 Gly2593Glu. In both blots, the four lanes on the far right of the figure depict blots where the tags were reversed on the constructs with controls of antibody specificity. An extended panel of control experiments together with overexposures for both blots are presented in Supplementary Figures 1 and 2 a

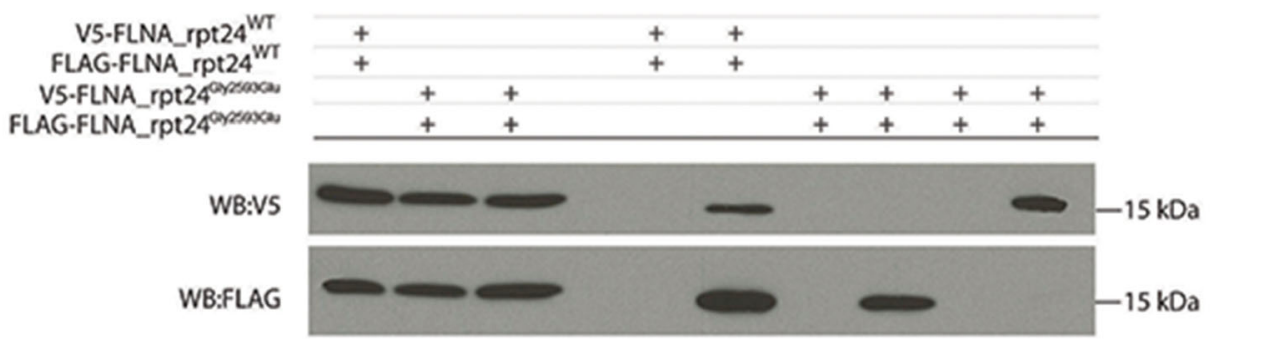

b

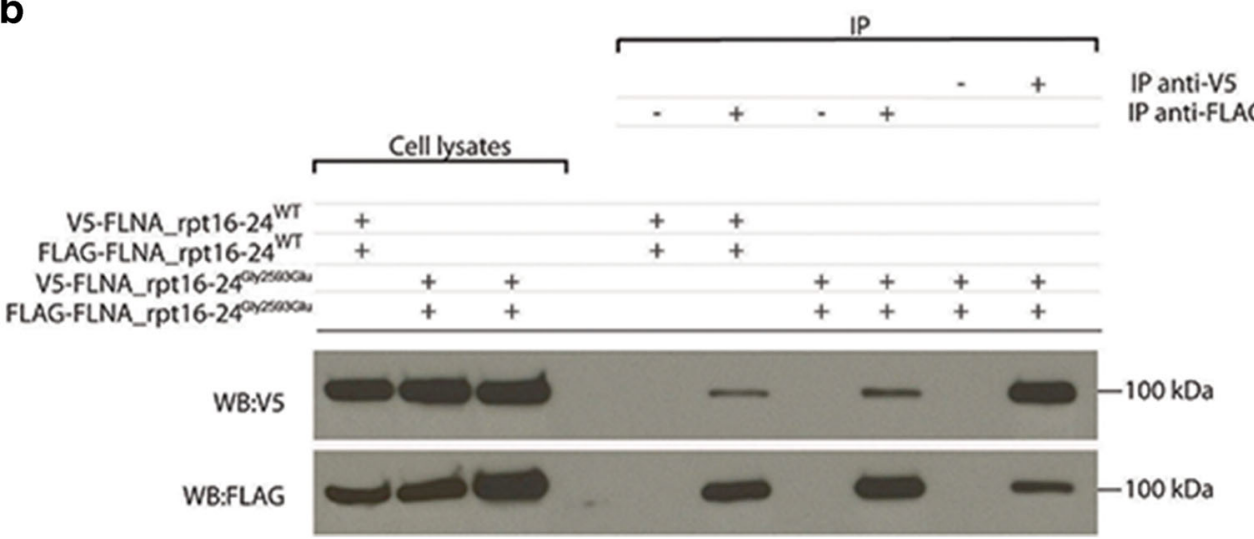


penetrant, consistent with the paucinodular presentation of another female with this same mutation [27] and the variability in intrafamilial expression of $\mathrm{PH}$ phenotypes described elsewhere $[4,23,27]$ although the potential remains for her to be clinically unaffected because she is also mosaic for this mutation [25].

Hemizygosity for loss of function mutations in FLNA typically leads to embryonic lethality in humans and mice [25, $26,32]$. The juxtaposition of such a mild clinical presentation with a germline mutation that is predicted to disrupt a proteinprotein interaction formerly held to be central to cellular function prompted a series of biochemical and cellular investigations to reconcile these observations. These data indicated that homodimerisation by FLNA repeat 24 is almost eliminated by the Gly2593Glu substitution. Studies using extended FLNA $^{\mathrm{G} 2593 \mathrm{E}}$ constructs, containing FLNA sequences from hinge 1 to repeats $16-24$, showed that these proteins have the ability to homodimerise via a mechanism that is not dependent on the canonical repeat 24 dimerisation interface. The mechanism of a compensatory dimerisation activity remains to be elucidated in detail although a previous study also suggested the existence of a second dimerisation site between repeat 16 to 23 using a yeast two-hybrid approach [33]. The implication of these observations is that the role of FLNA in sponsoring neuronal migration and cardiac and vascular morphogenesis whether it be through the sponsorship of cell autonomous neuronal migration [31], vesicle trafficking/cell adhesion [34] or the initiation of cellular migration [35] is substantially intact in individuals who are hemizygous for the Gly2593Glu substitution.

Expression studies utilising extended constructs containing the Gly2593Glu mutation showed that FLNA dimerises via rod 2 in the presence of this mutation. This region of FLNA adopts a complex tertiary structure, and the topology of this compensatory dimerisation site is unclear. The high structural similarity of repeats 17,19 and $21-23$ to repeat 24 presents the possibility for dimerisation via a similar mechanism demonstrated for repeat 24 [21] — the anti-parallel alignment of the two monomers. Such a configuration occurs between other actin binding proteins such as $\alpha$-actinin and $\beta$-spectrin [36] but would seem unlikely for FLNA since imaging studies have only detected V- and L-shaped structures that would preclude such a topology [20,37]. Alternatively, the accessory dimerisation interaction could be mediated by a second protein linking the two FLNA monomers. Potential candidates are the FLNA binding Rho-GTPase activating protein (FILGAP), a cell polarity and movement regulator [38] and Refilins A and B, which are homodimerising cross-linkers of FLNA in actin stress fibres [39]. FILGAP functions as a dimer, and a topographical model has suggested that FILGAP proteins interact with filamin repeat 23 and could serve to link FLNA monomers [40]. Efficient interaction of FILGAP with FLNA repeat 23 requires hinge 2 and repeat 24 structures to be intact [40], and therefore, it is unclear whether FILGAP alone could account for the accessory dimerisation activity demonstrated here where the repeat 24 components of each monomer are not apposed. Refilin B has FLNA binding sites within the rod domain 2 (repeats 16-23) [39] and could also mediate dimerisation of FLNA in the context of a repeat 24 domain that lacks a dimerisation interface.

Since the FLNA ${ }^{\mathrm{G} 2593 \mathrm{E}}$ substitution alters the dimerisation interface, which itself is apposed in an anti-parallel fashion to another monomer interface and therefore located remote from the solvent-exposed surface of the protein, this substitution is expected to have minimal effect on many protein binding sites that have been mapped to FLNA repeat 24 [9]. Over 70 proteins have been identified to interact with FLNA with the majority of proteins occurring near its C-terminus. Limited information is available regarding whether these interactions take place with monomeric or dimeric FLNA. If such interactions require the formation of a homodimeric repeat 24 for binding, these interactions could be affected in a cell harbouring FLNA ${ }^{\mathrm{G} 2593 \mathrm{E}}$. If this is the case, the mildness of the clinical phenotype perhaps indicates that for many of these interactions, the clinical consequences of their disruption is either minimal or that there is no pre-requisite for filamin to form a dimer via repeat 24 for the interactions to exert their physiological functions.

This study on the pathogenesis of $\mathrm{PH}$ caused by mutations in the $3^{\prime}$ of FLNA has confirmed the indispensability of repeat 24 as a whole to FLNA function. Despite this, some mutations can confer survivable phenotypes in males, although the specific effects of mutations vary considerably and may include male phenotypes with minimal manifestations within the central nervous system.

Acknowledgments We are grateful for the willing participation of the family described here. This work was supported by Curekids New Zealand (SR) and the Royal Society of New Zealand Marsden Fund (AJSS and SR).

Conflict of interest The authors declare no conflicts of interest.

\section{References}

1. Fox JW, Lamperti ED, Eksioglu YZ, Hong SE, Feng Y, Graham DA, Scheffer IE, Dobyns WB, Hirsch BA, Radtke RA et al (1998) Mutations in filamin 1 prevent migration of cerebral cortical neurons in human periventricular heterotopia. Neuron 21:1315-1325

2. Kyndt F, Gueffet JP, Probst V, Jaafar P, Legendre A, Le Bouffant F, Toquet C, Roy E, McGregor L, Lynch SA et al (2007) Mutations in the gene encoding filamin $\mathrm{A}$ as a cause for familial cardiac valvular dystrophy. Circulation 115:40-49

3. Robertson SP, Twigg SR, Sutherland-Smith AJ, Biancalana V, Gorlin RJ, Horn D, Kenwrick SJ, Kim CA, Morava E, Newbury-Ecob R et al (2003) Localized mutations in the gene encoding the cytoskeletal protein filamin A cause diverse malformations in humans. Nat Genet 33:487-491 
4. Sheen VL, Jansen A, Chen MH, Parrini E, Morgan T, Ravenscroft R, Ganesh V, Underwood T, Wiley J, Leventer R et al (2005) Filamin A mutations cause periventricular heterotopia with Ehlers-Danlos syndrome. Neurology 64:254-262

5. Sun Y, Almomani R, Aten E, Celli J, van der Heijden J, Venselaar H, Robertson SP, Baroncini A, Franco B, Basel-Vanagaite L et al (2010) Terminal osseous dysplasia is caused by a single recurrent mutation in the FLNA gene. Am J Hum Genet 87:146-153

6. Hartwig JH, Tyler J, Stossel TP (1980) Actin-binding protein promotes the bipolar and perpendicular branching of actin filaments. J Cell Biol 87:841-848

7. Gehler S, Baldassarre M, Lad Y, Leight JL, Wozniak MA, Riching KM, Eliceiri KW, Weaver VM, Calderwood DA, Keely PJ (2009) Filamin A-beta1 integrin complex tunes epithelial cell response to matrix tension. Mol Biol Cell 20:3224-3238

8. Lynch CD, Gauthier NC, Biais N, Lazar AM, Roca-Cusachs P, Yu CH, Sheetz MP (2011) Filamin depletion blocks endoplasmic spreading and destabilizes force-bearing adhesions. Mol Biol Cell 22:12631273

9. Nakamura F, Stossel TP, Hartwig JH (2011) The filamins: organizers of cell structure and function. Cell Adhes Migr 5

10. Fucini P, Renner C, Herberhold C, Noegel AA, Holak TA (1997) The repeating segments of the F-actin cross-linking gelation factor (ABP120) have an immunoglobulin-like fold. Nat Struct Biol 4:223-230

11. Gorlin JB, Yamin R, Egan S, Stewart M, Stossel TP, Kwiatkowski DJ, Hartwig JH (1990) Human endothelial actin-binding protein (ABP-280, nonmuscle filamin): a molecular leaf spring. J Cell Biol 111:1089-1105

12. Gardel ML, Nakamura F, Hartwig JH, Crocker JC, Stossel TP, Weitz DA (2006) Prestressed F-actin networks cross-linked by hinged filamins replicate mechanical properties of cells. Proc Natl Acad Sci U S A 103:1762-1767

13. Himmel M, Van Der Ven PF, Stocklein W, Furst DO (2003) The limits of promiscuity: isoform-specific dimerization of filamins. Biochemistry 42:430-439

14. Weihing RR (1988) Actin-binding and dimerization domains of HeLa cell filamin. Biochemistry 27:1865-1869

15. Pudas R, Kiema TR, Butler PJ, Stewart M, Ylanne J (2005) Structural basis for vertebrate filamin dimerization. Structure 13:111-119

16. Kainulainen T, Pender A, D'Addario M, Feng Y, Lekic P, McCulloch CA (2002) Cell death and mechanoprotection by filamin a in connective tissues after challenge by applied tensile forces. J Biol Chem 277:21998-22009

17. Kasza KE, Nakamura F, Hu S, Kollmannsberger P, Bonakdar N, Fabry B, Stossel TP, Wang N, Weitz DA (2009) Filamin A is essential for active cell stiffening but not passive stiffening under external force. Biophys J 96:4326-4335

18. Kesner BA, Ding F, Temple BR, Dokholyan NV (2010) N-terminal strands of filamin Ig domains act as a conformational switch under biological forces. Proteins 78:12-24

19. Shifrin Y, Arora PD, Ohta Y, Calderwood DA, McCulloch CA (2009) The role of FilGAP-filamin A interactions in mechanoprotection. Mol Biol Cell 20:1269-1279

20. Nakamura F, Osborn TM, Hartemink CA, Hartwig JH, Stossel TP (2007) Structural basis of filamin A functions. J Cell Biol 179:10111025

21. Seo MD, Seok SH, Im H, Kwon AR, Lee SJ, Kim HR, Cho Y, Park D, Lee BJ (2009) Crystal structure of the dimerization domain of human filamin A. Proteins 75:258-263

22. Gargiulo A, Auricchio R, Barone MV, Cotugno G, Reardon W, Milla PJ, Ballabio A, Ciccodicola A, Auricchio A (2007) Filamin A is mutated in $\mathrm{X}$-linked chronic idiopathic intestinal pseudoobstruction with central nervous system involvement. Am J Hum Genet 80:751-758

23. Guerrini R, Mei D, Sisodiya S, Sicca F, Harding B, Takahashi Y, Dorn T, Yoshida A, Campistol J, Kramer G et al (2004) Germline and mosaic mutations of FLN1 in men with periventricular heterotopia. Neurology 63:51-56

24. Hehr U, Hehr A, Uyanik G, Phelan E, Winkler J, Reardon W (2006) A filamin A splice mutation resulting in a syndrome of facial dysmorphism, periventricular nodular heterotopia, and severe constipation reminiscent of cerebro-fronto-facial syndrome. J Med Genet 43:541-544

25. Parrini E, Mei D, Wright M, Dorn T, Guerrini R (2004) Mosaic mutations of the FLN1 gene cause a mild phenotype in patients with periventricular heterotopia. Neurogenetics 5:191-196

26. Sheen VL, Dixon PH, Fox JW, Hong SE, Kinton L, Sisodiya SM, Duncan JS, Dubeau F, Scheffer IE, Schachter SC et al (2001) Mutations in the X-linked filamin 1 gene cause periventricular nodular heterotopia in males as well as in females. Hum Mol Genet 10: $1775-1783$

27. Fergelot P, Coupry I, Rooryck C, Deforges J, Maurat E, Sole G, Boute O, Dieux-Coeslier A, David A, Marchal C et al (2012) Atypical male and female presentations of FLNA-related periventricular nodular heterotopia. Eur J Med Genet 55:313-318

28. Liang CC, Park AY, Guan JL (2007) In vitro scratch assay: a convenient and inexpensive method for analysis of cell migration in vitro. Nat Protoc 2:329-333

29. Collins TJ (2007) ImageJ for microscopy. Biotechniques 43:25-30

30. Reinstein E, Frentz S, Morgan T, Garcia-Minaur S, Leventer RJ, McGillivray G, Pariani M, van der Steen A, Pope M, HolderEspinasse $\mathrm{M}$ et al (2013) Vascular and connective tissue anomalies associated with $\mathrm{X}$-linked periventricular heterotopia due to mutations in filamin A. Eur J Hum Genet 21:494-502

31. Cunningham CC, Gorlin JB, Kwiatkowski DJ, Hartwig JH, Janmey PA, Byers HR, Stossel TP (1992) Actin-binding protein requirement for cortical stability and efficient locomotion. Science 255:325-327

32. Feng Y, Chen MH, Moskowitz IP, Mendonza AM, Vidali L, Nakamura F, Kwiatkowski DJ, Walsh CA (2006) Filamin A (FLNA) is required for cell-cell contact in vascular development and cardiac morphogenesis. Proc Natl Acad Sci U S A 103:19836-19841

33. Sheen VL, Feng Y, Graham D, Takafuta T, Shapiro SS, Walsh CA (2002) Filamin A and filamin B are co-expressed within neurons during periods of neuronal migration and can physically interact. Hum Mol Genet 11:2845-2854

34. Ferland RJ, Batiz LF, Neal J, Lian G, Bundock E, Lu J, Hsiao YC, Diamond R, Mei D, Banham AH et al (2009) Disruption of neural progenitors along the ventricular and subventricular zones in periventricular heterotopia. Hum Mol Genet 18:497-516

35. Baldassarre M, Razinia Z, Burande CF, Lamsoul I, Lutz PG, Calderwood DA (2009) Filamins regulate cell spreading and initiation of cell migration. PLoS One 4:e7830

36. Ylanne J, Scheffzek K, Young P, Saraste M (2001) Crystal structure of the alpha-actinin rod reveals an extensive torsional twist. Structure 9:597-604

37. Stossel TP, Condeelis J, Cooley L, Hartwig JH, Noegel A, Schleicher M, Shapiro SS (2001) Filamins as integrators of cell mechanics and signalling. Nat Rev Mol Cell Biol 2:138-145

38. Ohta Y, Hartwig JH, Stossel TP (2006) FilGAP, a Rho- and ROCKregulated GAP for Rac binds filamin A to control actin remodelling. Nat Cell Biol 8:803-814

39. Gay O, Gilquin B, Nakamura F, Jenkins ZA, McCartney R, Krakow D, Deshiere A, Assard N, Hartwig JH, Robertson SP et al (2011) RefilinB (FAM101B) targets filamin A to organize perinuclear actin networks and regulates nuclear shape. Proc Natl Acad Sci U S A 108: 11464-11469

40. Nakamura F, Heikkinen O, Pentikainen OT, Osborn TM, Kasza KE, Weitz DA, Kupiainen O, Permi P, Kilpelainen I, Ylanne J et al (2009) Molecular basis of filamin A-FilGAP interaction and its impairment in congenital disorders associated with filamin A mutations. PLoS One 4:e4928 